\title{
Spitzoid Neoplasms: Suggestions from Genomic Aberrations
}

\author{
Carmelo Urso \\ Dermatopathology Study Center of Florence, Florence, Italy
}

In the last decade, numerous studies have been performed in order to characterize the genetic aberrations of spitzoid neoplasms, with the goal of finding new biomarkers and, possibly, explaining the complex biology of these tumors [1]. In particular, fluorescence in situ hybridization assay, with 4 (11q13, 6p25, 6p11.1, 6q23), 6 (11q13, 6p25, 6p11.1, 6q23, 9p21, 9q11.1) or 5 (11q13, 6p25, 9p21, 9q11.1, 8q24) probes, and array comparative genomic hybridization have been proposed for diagnostic purposes. Unfortunately, these sophisticated techniques have appeared capable of separating tumors readily classifiable as benign or malignant at histologic examination, but they are "not very useful" in diagnosing histologically ambiguous or uncertain spitzoid neoplasms [1]. Some genetic aberrations, such as p-TERT mutation and BRAF fusion, seem to indicate a high risk and an unfavorable clinical course, but, at present, only few cases have been studied, and results need to be confirmed [2-4]. Moreover, some genetic alterations apparently characteristic of spitzoid neoplasms, such as HRAS mutation or ALK, ROS1, NTRK1, RET, MET, and NTRK3 fusions [1, 5], do not seem employable in the diagnosis, because they are not useful to separate benign from malignant cases.

However, if results from genetic studies are not presently utilizable for solving the diagnostic problems of spitzoid neoplasms, they seem to highlight 2 points that may be relevant: (1) spitzoid neoplasms show characteristic genomic aberrations (HRAS mutations; ALK, ROS1, NTRK1, RET, MET, and NTRK3 fusions) different from those characterizing other classes or groups of melanocytic lesions, such as common and congenital nevi (which harbor BRAF and NRAS mutations), blue melanocytic lesions (which harbor GNAQ and GNA11 mutations), nonspitzoid melanomas (which harbor BRAF, HRAS, NRAS, KRAS, NF1, and KIT mutations) [1], and deep penetrating nevi/tumors (which harbor $\beta$-catenin and MAPK pathway mutations) [6]; (2) all spitzoid neoplasms, including lesions currently labeled as Spitz nevus, 

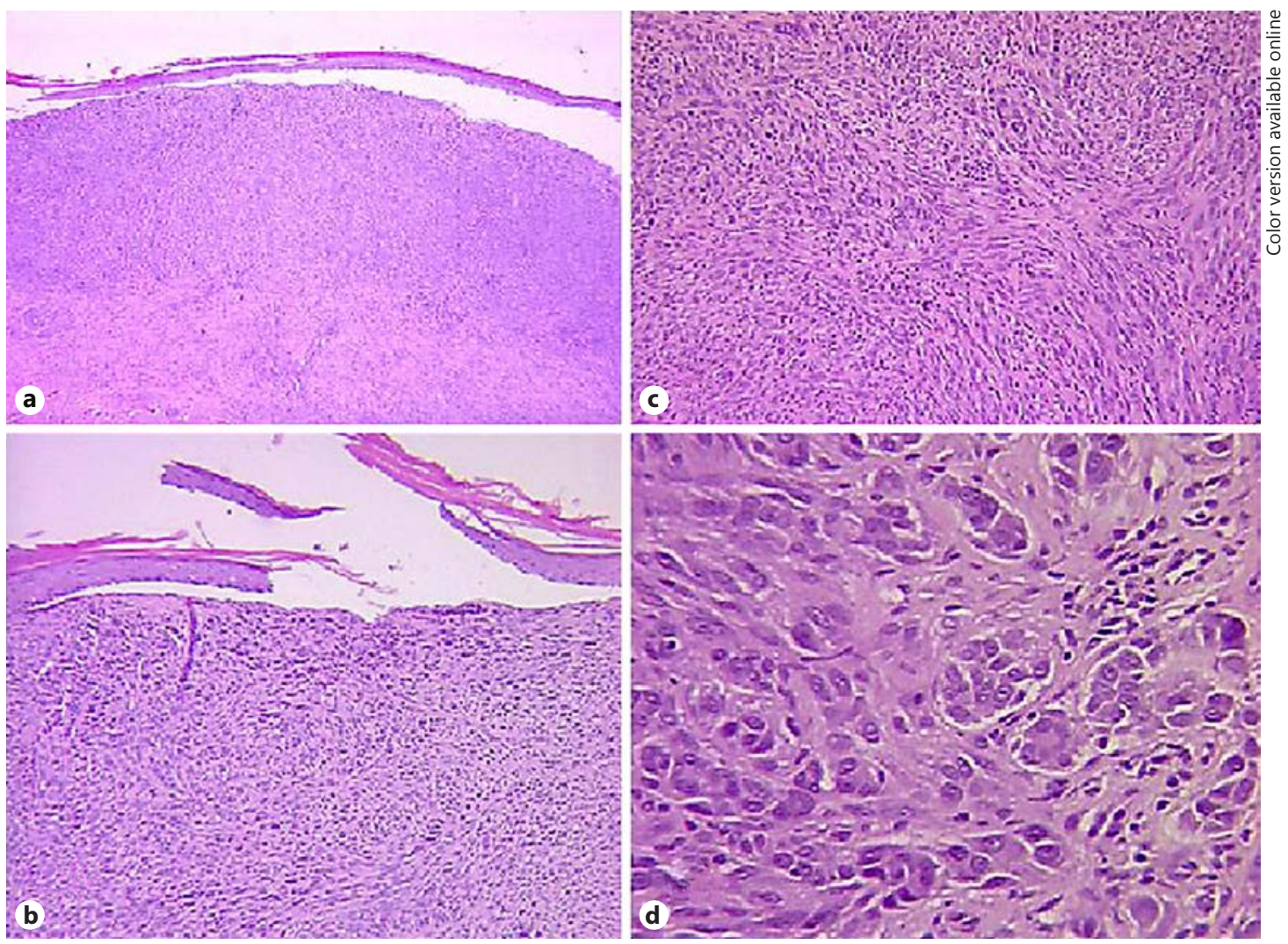

Fig. 1. Spitzoid neoplasm in a 38-year-old female patient; the lesion, located on the right thigh and measuring $7 \mathrm{~mm}$, was diagnosed as "atypical Spitz tumor"; the sentinel node was negative; the patient presented no adverse events at follow-up (44 months). a, b The melanocytic proliferation was located in the dermis; the lesion showed consumption of the epidermis and extensive subepidermal clefts (hematoxylin and eosin stain; original magnification $\times 75$ and $\times 175$, respectively). c, $\mathbf{d}$ Tumor cells, spindle and epithelioid in shape, formed solid sheets, appearing moderately atypical (hematoxylin and eosin stain; original magnification $\times 175$ and $\times 400$, respectively).

atypical Spitz tumor, and spitzoid melanoma, basically share the same chromosomal aberrations [1].

It is inevitable to note that both of these points tend to weaken some classical and current concepts on spitzoid neoplasms. The first point weakens the widely accepted concept that spitzoid neoplasms are mere morphologic variants of benign and malignant melanocytic neoplasms, i.e., Spitz nevus, a variant of nevus; spitzoid melanoma, a variant of melanoma. In fact, the characteristic genomic aberrations found in spitzoid neoplasms clearly seem to set this class of melanocytic tumors apart from all other melanocytic lesions: spitzoid neoplasms appear as an autonomous and separate class or group of tumors, as previously noted in the old and recent literature $[7,8]$. The second point casts serious doubt on the real possibility that spitzoid neoplasms are constituted by several (3 or 4) structurally and biologically different (benign, borderline, and malignant) tumors. In fact, the lack of genomic differences suggests spitzoid neoplasms to be a homogeneous group of tumors, although with variable biologic behaviors. In this context, labels, such as "Spitz nevus," "atypical Spitz nevus," "atypical Spitz tumor," and "spitzoid melanoma," would seem to represent prognostic categories rather than specific diagnoses [9] (Fig. 1,2). In this case, these tumors could also appear 

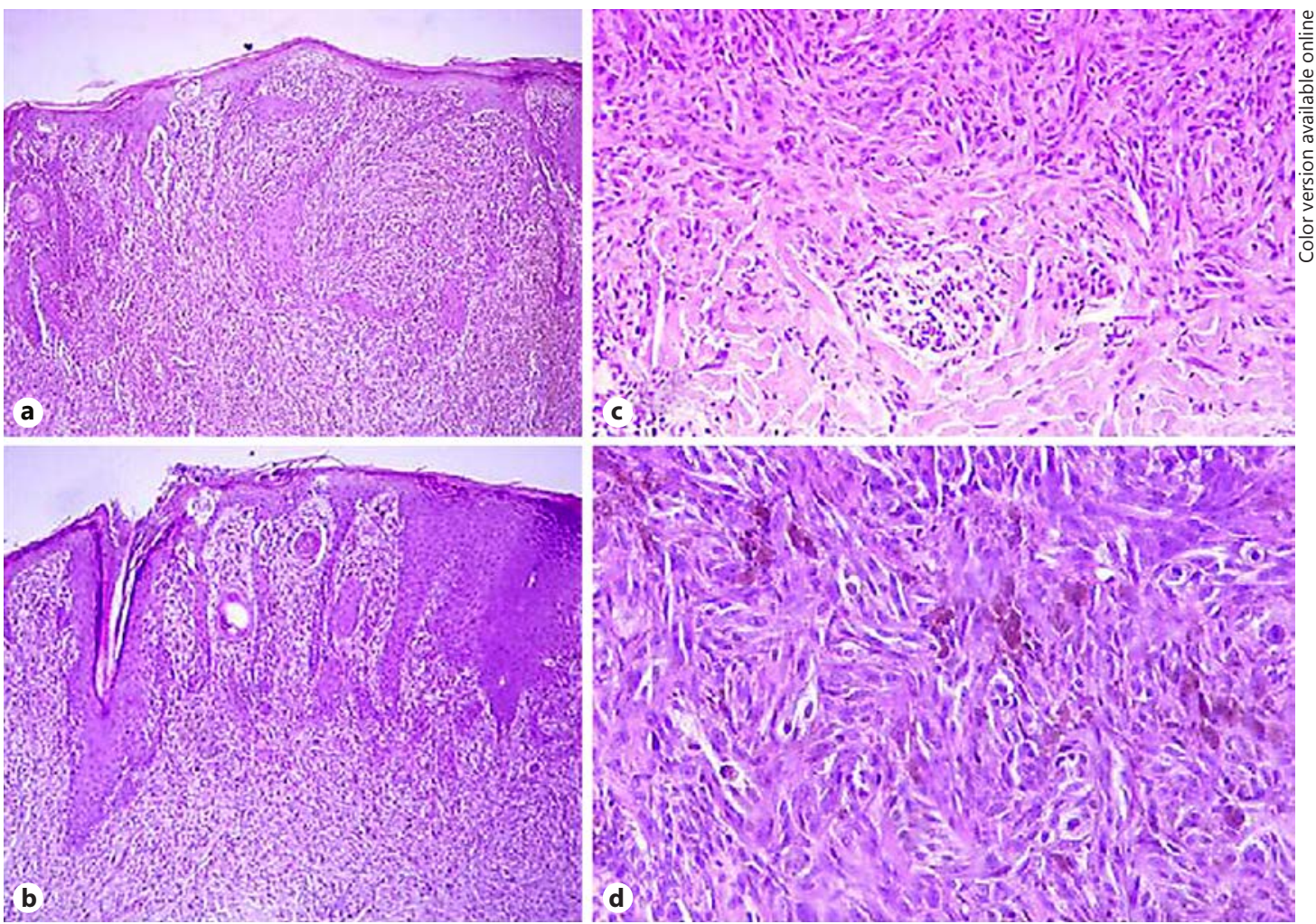

Fig. 2. Spitzoid neoplasm in a 17-year-old female patient; the lesion, located on the right thigh and measuring $6 \mathrm{~mm}$, was diagnosed as "atypical Spitz tumor"; 28 months after the diagnosis, a metastatic inguinal lymph node was detected; after lymphadenectomy, 4 additional lymph nodes were found to be involved. a, b The melanocytic proliferation was located in the dermis, with focal involvement of a hyperplastic epidermis, showing hypergranulosis (hematoxylin and eosin stain; original magnification $\times 100$ and $\times 175$, respectively). c Spindle tumor cells appeared moderately atypical and showed incomplete maturation (hematoxylin and eosin stain; original magnification $\times 300$ ). $\mathbf{d}$ Solid sheets, scanty melanin pigment and mitoses (hematoxylin and eosin stain; original magnification $\times 400$ ).

as a unique entity (Spitz tumor). This unique neoplasm would not be benign or borderline but would possess a certain malignant potential, expressible as statistical probability that an adverse event (nodal or visceral metastasis, death) occurs or is detected. This malignant potential would appear to be low, statistically lower than expected in conventional melanoma of the same thickness [9], and variable, ranging in different single cases from very low (tending to 0 , but greater than 0 ) to high (aggressive) [10].

\section{Statement of Ethics}

The author has no ethical conflicts to disclose.

\section{Disclosure Statement}

The author declares no funding sources for the work, nor conflicts of interests. 


\section{References}

1 Wiesner T, Kutzner H, Cerroni L, Mihm MC, Busam KJ, Murali R: Genomic aberrations in spitzoid melanocytic tumours and their implications for diagnosis, prognosis and therapy. Pathology 2016;48:113-131.

-2 Lee S, Barnhill RL, Dummer R, Dalton J, Wu J, Pappo A, Bahrami A: TERT promoter mutations are predictive of aggressive clinical behavior in patients with spitzoid melanocytic neoplasms. Sci Rep 2015;5:11200.

-3 Amin SM, Haugh AM, Lee CY, Zhang B, Bubley JA, Merkel EA, Verzì AE, Gerami P: A comparison of morphologic and molecular features of BRAF, ALK, and NTRK1 fusion spitzoid neoplasms. Am J Surg Pathol 2017;41:491498.

4 Requena C, Heidenreich B, Kumar R, Nagore E: TERT promoter mutations are not always associated with poor prognosis in atypical spitzoid tumors. Pigment Cell Melanoma Res 2017;30:265-268.

$>5$ Yeh I, Tee MK, Botton T, Shain AH, Sparatta AJ, Gagnon A, Vemula SS, Garrido MC, Nakamaru K, Isoyama T, McCalmont TH, LeBoit PE, Bastian BC: NTRK3 in Spitz tumors. J Pathol 2016;240:282-290.

-6 Yeh I, Lang UE, Durieux E, Tee MK, Jorapur A, Shain AH, Haddad V, Pissaloux D, Chen X, Cerroni L, Judson RL, LeBoit PE, McCalmont TH, Bastian BC, de la Fouchardiere A: Combined activation of MAP kinase pathway and $\beta$-catenin signaling cause deep penetrating nevi. Nat Commun 2017;8:644.

-7 McWorther HE, Woolner LB: Pigmented nevi, juvenile melanomas, and malignant melanomas in children. Cancer 1954;7:564-585.

8 Urso C: A new perspective for Spitz tumors? Am J Dermatopathol 2005;27:364-366.

-9 Cerroni L, Barnhill R, Elder D, Gottlieb G, Heenan P, Kutzner H, LeBoit PE, Mihm M Jr, Rosai J, Kerl H: Melanocytic tumors of uncertain malignant potential. Results of a tutorial held at the XXIX Symposium of the International Society of Dermatopathology in Graz, October 2008. Am J Surg Pathol 2010;34:314-326.

10 Urso C: Time to reconsider Spitzoid neoplasms? Dermatol Pract Concept 2016;6:8. 